\title{
The effect of various dominant VRN alleles and their combinations on the duration of development phases and productivity in common wheat lines
}

\author{
Chumanova E.V. ${ }^{1 *}$, Efremova T.T. ${ }^{1}$, Kruchinina Y.V. ${ }^{1,2}$ \\ ${ }^{1}$ Institute of Cytology and Genetics, SB RAS, Novosibirsk, Russia \\ ${ }^{2}$ Novosibirsk State Agrarian University, Novosibirsk, Russia \\ *e-mail: chumanova@bionet.nsc.ru
}

Establishing the effect of different alleles of the $V R N$ loci and their combinations on the duration of the developmental phases and the productivity of common wheat is of immediate practical importance for breeding. Since most varieties of Russia and Western Siberia carry the dominant alleles of $V r n-A 1$ and $V r n-B 1$ genes, were obtained two lines of winter cultivar Bezostaya 1 (Bez1) with the combination of alleles of the $V R N-1$ locus: Bez1Vrn-Ala Vrn-Bla and Bez1Vrn-Ala Vrn-B1c. Homozygous plants were isolated in the $\mathrm{F}_{2}$ generation using allele-specific primers for the $V R N-A 1$ and $V R N-B 1$ loci. Based on the genetic segregation of $\mathrm{F}_{2}$ hybrids with tester isogenic lines, it was confirmed that the obtained lines carry two dominant genes: Vrn-Al and Vrn-B1. The presence of the Ppd-Dla allele in lines of cultivar Bez1 was shown with the use of PCR-marker. It was established that the lines with two dominant alleles headed on day 40, which was 2, 8 and 5 days shorter than in the isogenic lines i:Bez1Vrn-Ala, i:Bez1Vrn-Bla and $\mathrm{i}:$ Bez1Vrn-B1c, respectively ( $p \leq 0.01-0.001)$. Also, these lines have reduced the period of "tillering-first node" compared to the above lines by 2, 9, and 8 days, respectively $(p<0.001)$. The study of the dynamics of the growth cone in the lines of the Bez1 and Sava cultivars showed that the differences began to appear from the "tillering-first node" stage. The lines with the dominant allele Vrn-Ala were ahead of other lines at the III-IV stages of organogenesis in the degree of differentiation and the size of the growth cone and the lines with the Vrn-Blc allele were ahead the lines with the Vrn-Bla allele. It was found that the lines with dominant allele $V r n-B l c$ were the most productive, and the Bez1Vrn-Ala Vrn-B1c line was more productive than the Bez1Vrn-Ala Vrn-Bla. Acknowledgements: The work supported by the RFBR grant No. 18-34-00146 mol_a and Budget Project No. 0324-2019-0039. 